\title{
Propagation of ultrahigh-energy neutrinos through the Earth
}

\author{
R. Horvat \\ "Rudjer Bošković" Institute, P.O.Box 1016, 10001 Zagreb, Croatia
}

\begin{abstract}
The dispersion relation in matter of ultrahigh-energy neutrinos above the pole of the $W$ resonance $\left(E_{\nu} \gtrsim 10^{7} \mathrm{GeV}\right)$, is studied. We perform our calculation using the real-time formulation of Thermal Field Theory in which the massless limit for the $W$ boson is taken. The range of active-to-sterile neutrino oscillation parameters for which there is significant mixing enhancement during propagation through the interior of the Earth, and therefore significant attenuation of neutrino beams in the Earth at high energies, is estimated. Finally, this range is considered in view of the cosmological and astrophysical constraints.
\end{abstract}


It is now well established [1] that the Earth's diameter exceeds the attenuation length of neutrinos with energies greater than $25 \mathrm{TeV}$. Such an estimate was based on the calculation of the cross sections for $\nu N$ collisions at ultrahigh energies (UHE), $\left(E_{\nu} \gtrsim 1 \mathrm{TeV}\right)$. Because of the smallness of the electron mass, $\nu$ interactions are generally considered as negligible with respect to $\nu N$ interactions; $\nu N$ interactions therefore provide the dominant signal and account for most of the attenuation of neutrino beams in the interior of the Earth at ultrahigh energies [1,2]. There is one exception though, the resonant formation of the intermediate $W^{-}$boson in $\bar{\nu} e$ interactions in the neighborhood of $E_{\nu}^{r e s}=M_{W}^{2} / 2 m_{e} \simeq 6.3 \times 10^{15} \mathrm{eV}$.

The promising tool for detection of UHE cosmic neutrinos by means of neutrino telescopes [4] consists of recording the long-range muons produced in charged-current $\nu N$ interactions that occurs in matter surrounding the detector. Apart from efficient shielding from the flux of atmospheric muons, such upward-going muon events have the advantage of enhancing the effective volume in proportion to the range of the produced muons (typically a few kilometers for $E_{\mu} \simeq 10 \mathrm{TeV}$ ). For our purpose it is important to note that the rate for upwardgoing muons does not depend only on the probability for neutrino conversion to a muon with energy above the threshold energy, but also through the interaction length which is responsible for the attenuation of the neutrino flux due to interactions in the Earth's interior. The typical situation that occurs for $E_{\nu} \lesssim 10^{5} \mathrm{GeV}$ is that the upward rates depend little [1] on the calculated $\nu N$ cross sections, since the enhanced (weakened) interaction rate is nearly compensated by the enhanced (weakened) attenuation of UHE neutrinos propagating through the Earth. On the other hand, the detection of cosmic neutrinos at energies of $10^{16} \mathrm{eV}$ or larger is beset by the problem of the increased importance of attenuation of neutrino beams [1,2]. However, even so, the upward rates produced by neutrinos from powerful radiation sources, like Active Galactic Nuclei (AGN) [5], should be observable in a detector whose effective area is $A \simeq 0.1 \mathrm{~km}^{2}$.

As for event rates involving electron neutrinos, they are generally smaller than the muon event rate by the flux ratio (the initial fluxes of UHE neutrinos originating from AGNs are expected to have a ratio $\nu_{e} / \nu_{\mu} \simeq 1 / 2$ ) times the detector length divided by the mean muon range, because of the rapid energy loss of electrons (or annihilation for positrons). Still, it was shown recently [6] that the Landau-Pomeranchuk-Migdal effect [7] may effectively enhance the electron range by detecting upward-going air showers initiated by the $\nu_{e}$ interaction near the Earth's surface. On the other hand, resonant $\bar{\nu} e$ scattering contributes significantly to the attenuation of $\bar{\nu}_{e}$ 's, meaning that the flux of electron antineutrinos in the range $2 \times 10^{15} \mathrm{eV} \leq E_{\nu} \leq 2 \times 10^{16} \mathrm{eV}$ is extinguished for neutrinos traversing the Earth [1,2].

In the present paper we are going to consider another mechanism for attenuation of UHE neutrinos propagating through the Earth, namely, matter enhanced neutrino oscillations $\nu_{e} \leftrightarrow \nu_{s}$, where $s$ is a sterile neutrino (e.g. a singlet under the gauge symmetry of the Standard model). We shall be concerned exclusively with the case of $\nu_{e} \leftrightarrow \nu_{s}$ oscillations where the energy of UHE neutrinos is above the resonant energy, $E_{\nu} \gtrsim E_{\nu}^{r e s}$. Owing to the new form of the effective matter potential in this regime, we are in position to study MSW resonance effect [8] previously ignored in the literature. On the other hand, the matter effect of the Earth through the standard effective potentials $\left(E_{\nu}<<E_{\nu}^{r e s}\right)$, in the region of oscillation parameters relevant for the solar and atmospheric neutrinos as well as those suggested by the LSND result, is well established now [9]. Even more, a new effect of matter-enhanced neutrino mixing, based on a maximal constructive interference among 
transition amplitudes, has been discovered recently [10]. In the following, we shall first derive the induced mass squared of the electron neutrino with $E_{\nu} \gtrsim E_{\nu}^{r e s}$, then we find the range of neutrino parameters for which there is matter-enhanced $\nu_{e} \leftrightarrow \nu_{s}$ oscillation during propagation of $\nu_{e}$ through the Earth and finally we discuss if the established range could survive constraints from type II supernovae as well as big bang nucleosynthesis on $\nu_{e} \leftrightarrow \nu_{s}$ mixing.

Effects of a medium on neutrino propagation is determined by the difference of potentials, whose standard-model contribution in the context of Thermal Field Theory (TFT) may readily be obtained from the relevant thermal self-energies of a neutrino: charged-current, neutral-current and tadpole. Of these, only the charged-current and tadpole contribution are relevant for our consideration. Let us consider the charged-current diagram in more detail. Since the "target" electrons are massive, the $W$ boson may be considered "massless" always when $s \simeq 2 E_{\nu} m_{e} \gg M_{W}^{2}$. This corresponds to energies in the lab frame $E_{\nu} \gtrsim 6 \times 10^{15} \mathrm{eV}$. In the opposite limit, $s \ll M_{W}^{2}$, the $W$ boson should be considered "massive" and the usual contact approximation for the $W$-propagator is adequate. Using the real-time formulation of TFT, we discover by explicit calculation that the induced mass squared of $\nu_{e}$ is equivalent to the fermion thermal mass squared [11],

$$
A_{\nu}^{c c}=\frac{g^{2}}{2 \pi^{2}} \int_{0}^{\infty} k d k n_{e}\left(k_{0}\right) \quad\left(E_{\nu} \gg E_{\nu}^{r e s}\right),
$$

where $g \simeq 0.63$ is the gauge coupling constant. Note that in contrast to the mass squared induced by the standard MSW potential,

$$
A_{\nu}^{c c}=2 \sqrt{2} G_{F} N_{e} E_{\nu} \quad\left(E_{\nu} \ll E_{\nu}^{r e s}\right),
$$

(1) is independent of neutrino energy and also there is no explicit dependence on the number density of electrons. It should be clearly stated here that actually we are not dealing with field theory in equilibrium since all the electrons in the medium are bound electrons. Still, one is allowed to retain the usual real-time formalism by taking the (11)-component of the electron propagator to be

$$
S_{11}(k)=\left(\not k+m_{e}\right)\left(\frac{1}{k^{2}-m_{e}^{2}+i \epsilon}+2 \pi i n_{e}\left(k_{0}\right) \delta\left(k^{2}-m_{e}^{2}\right)\right),
$$

where now a bound electron is assigned a distribution $n_{e}\left(k_{0}\right)$. Thus (1) describes the planewave impulse approximation, which is, for instance, the basic approximation of electron momentum spectroscopy of atoms and molecules [12].

Although a connection with equilibrium TFT is now established, one may still wonder why (1) is equivalent to the fermion thermal mass, that is, to the characteristic mass scale which appears naturally only in the high-temperature limit of the fermion self-energy. Before going into details, let us stress that to first order in perturbation theory at high temperature, the poles of the full fermion propagator are determined just by the thermal mass [11]. In hot theories, the high-temperature limit means that the temperature is much larger than mass of the particles under consideration $\left(m_{e}, M_{W}\right.$ in our case) and the external momenta. Consequently, all particle masses can be ignored for practical purposes. In the standard electro-weak theory we now show that the opposite case, when the external momenta are 
large and the characteristic scale of the medium is much less than the particle masses, can faithfully mimic the high-temperature limit. Indeed, when $E_{\nu} \gg E_{\nu}^{r e s}$, the four-momentum squared of the $W$ boson is always much larger than $M_{W}^{2}$, and hence the $W$ boson can be considered "massless". To get rid of the electron mass, note that it appears in the numerator of the thermal part of the electron propagator, and both in the numerator and denominator of the vacuum part of $S_{11}$ [see Eq. (3)]. It is however trivially to see that $m_{e}$ from the numerator disappears when sandwiched between the two electroweak vertices $\gamma_{\mu} L$, where $L=\frac{1}{2}\left(1-\gamma_{5}\right)$ is the projection operator for the left-chiral fermions. On the other hand, the real part of the neutrino self-energy is given only by a contribution where a cut is through the electron line, since $W$ bosons are absent from the medium. This means that there is no contribution from the vacuum part of $S_{11}$, where $m_{e}$ appears in the denominator . This completes the proof that the high-energy limit is equivalent to the high-temperature limit of hot gauge theories. Strictly speaking, since the thermal mass always involves the thermal effects from both the $W$ boson and the electron propagators, the only difference is that the former is absent in (1).

Using the above interpretation for bound states and keeping the same normalization as for quasifree states, one can rewrite (1) in the following form,

$$
A_{\nu}^{c c} \simeq 0.2<k^{-1}>N_{e},
$$

where $\langle k>$ is the average momentum of bound electrons. For the rough estimates presented here, it is sufficient to assume that $\left\langle k^{-1}\right\rangle \simeq\langle k\rangle^{-1}$. Let us choose the average momentum per atom (with the atomic number $Z$ ) as a quantity of interest here. Going back to atomic physics, one can determine this quantity by applying the Thomas-Fermi method [13] to the calculation of the total ionization energy of a neutral atom. The result is

$$
<k>^{Z} \simeq 4.6 Z^{2 / 3} \mathrm{keV} .
$$

For our purpose, let us recall that the interior of the Earth consists of two regions of slowly varying density - the core and the mantle, with particularly strong density change between the lower mantle and outer core. The density profile of the Earth can be found in [14]. The density of the mantle increases from 3 to $5.5 \mathrm{gcm}^{-3}$ (the average value is $4.7 \mathrm{gcm}^{-3}$ and the average electron fraction is 0.49 ), while the density of the core varies from 10 to $13 \mathrm{gcm}^{-3}$ (the average value is $11.8 \mathrm{gcm}^{-3}$ and the average electron fraction is 0.47 ). The core comprises heavier elements, presumably nickel $(Z=28)$. Hence from $(5)$ we have

$$
<k>_{\text {core }}^{Z} \simeq 42 \mathrm{keV} .
$$

The mantle consists of lighter elements $(Z=8-16)$, and our estimate in this case is

$$
<k>_{\text {mantle }}^{Z} \simeq 25 \mathrm{keV} .
$$

Before determination from the resonance condition of a range of neutrino masses where maximum mixing enhancement may occur, one should consider the tadpole graph as well. Being a constant independent of the external neutrino momentum, it is the same as in the standard MSW case $\left(E_{\nu} \ll E_{\nu}^{r e s}\right)$, giving rise to the induced mass squared which grows linearly with energy, i.e., 


$$
A_{\nu}^{\text {tadpole }}=-\sqrt{2} G_{F} N_{n} E_{\nu},
$$

where $N_{n}$ is the neutron number density. Apart from a negative sign in (8), let us compare the magnitude of (8) with the charged-current contribution (1). It turns out that for $E_{\nu} \gtrsim 10^{9} \mathrm{GeV}$ (8) is beginning to dominate over (1). For our purpose, it is however enough to consider $E_{\nu} \simeq 10^{7}-10^{8} \mathrm{GeV}$ since the short $\nu_{e}$ interaction length for energies $E_{\nu}>10^{9} \mathrm{GeV}$ means that the flux of electron neutrinos is extinguished for neutrinos traversing the Earth. Hence for $E_{\nu} \lesssim 10^{9} \mathrm{GeV}$, the total induced mass squared for $\nu_{e}$ is essentially given by (1). It is interesting to note that at a particular energy around $10^{9} \mathrm{GeV}$ there is a nearly complete cancellation of matter effects in the neutrino propagator due to the sign of (8).

With the above simplifications and the range of densities in the Earth's interior as discussed before, one finds from the resonance condition, $A_{\nu}^{c c} \approx \Delta m_{e s}^{2}$, a range of neutrino masses where maximal mixing enhancement may occur,

$$
0.07<\Delta m_{e s}^{2} / k e V^{2}<0.12 \quad\left(10^{9} \mathrm{GeV} \gtrsim E_{\nu} \gtrsim 10^{7} \mathrm{GeV}\right) .
$$

Here we have taken a small mixing angle, $\cos 2 \theta \approx 1$, in order to study oscillation enhancement. $\Delta m_{e s}^{2}>0$ in (9) means that $\nu_{s}$ is heavier than $\nu_{e}$. This is also true for $\bar{\nu}_{e} \leftrightarrow \bar{\nu}_{s}$ oscillations as $A_{\bar{\nu}}^{c c}>0$ (in contrast to the standard MSW potential where the sign is reversed for antineutrinos).

Notice that because of the resonance condition which is energy independent and the range of densities in the Earth, the range (9) is actually very small. Even so, it lies in the region which might be very interesting to astrophysics as well as cosmology. We recall that the prediction for the sterile neutrino of $\sim \mathrm{keV}$ mass is not in contradiction with any of the present bound. Indeed, the $\sim \mathrm{keV}$ mass is needed if active-to-sterile neutrino oscillations are to solve the pulsar velocity puzzle [15]. In contrast to active-to-active oscillations, this solution is not in conflict with the cosmological bound on stable neutrino masses since the $\sim \mathrm{keV}$ mass sterile neutrino has been proposed as a viable dark-matter candidate [16].

It is well known that if we add the reported results from the LSND collaboration [17 to the list of neutrino anomalies, then the explanation of all of them requires a four-neutrino scheme with three active neutrinos $\nu_{e}, \nu_{\mu}, \nu_{\tau}$ and one electroweak-singlet neutrino [18]. This introduces the 'LSND gap' of a few eV, a few orders of magnitude below our preferred range (9). However, the LSND result is not confirmed (although not completely ruled out) by a similar KARMEN experiment [19].

It is easy to estimate the range of active-to-sterile neutrino parameters for which there is significant enhancement mixing during propagation through the Earth. For significant transitions to developed, it is necessary that the propagation distance be greater than about a quarter of a wavelength at resonance [20]. This constraint gives us a lower limit on the mixing angle. Taking the longest distance through the Earth $\left(2 R_{\text {earth }}\right)$ we have,

$$
\frac{\pi E_{\nu}}{\Delta m_{e s}^{2} \sin 2 \theta}<2 R_{\text {earth }},
$$

which for $E_{\nu}=10^{7} \mathrm{GeV}$ and $2 R_{\text {earth }}=1.27 \times 10^{9} \mathrm{~cm}$ gives $(\sin 2 \theta)_{\text {core }}>2 \times 10^{-3}$ and $(\sin 2 \theta)_{\text {mantle }}>3.4 \times 10^{-3}$. 
There is however a stronger limit on $\sin 2 \theta$ coming from the condition for unsuppressed oscillations. The oscillation frequency must be real, otherwise the system is critically overdamped, the oscillations would be fully incoherent, and hence in fact there will be no oscillations. Since for neutrino energy in the range $10^{15} \mathrm{eV} \leq E_{\nu} \leq 10^{21} \mathrm{eV}$, the cross section scales with $E_{\nu}$ as $\sigma \propto E_{\nu}^{0.4}$ [1.:2], one finds the mean free path for neutrinos with $E_{\nu}=10^{7} \mathrm{GeV}$ to be $l=0.1 \times 2 R_{\text {earth }}$. The condition for unsuppressed oscillations,

$$
l_{m}<2 l,
$$

then gives $(\sin 2 \theta)_{\text {core }}>4 \times 10^{-2}$ and $(\sin 2 \theta)_{\text {mantle }}>7 \times 10^{-2}$. Notice that although the Earth is opaque to UHE neutrinos, the oscillations may proceed unsuppressed whenever the above requirement is satisfied.

Let us finally check up the range $\left(\Delta m_{e s}^{2} / k e V^{2} \simeq 0.12, \sin 2 \theta>4 \times 10^{-2}\right)$ from a viewpoint of astrophysics and cosmology. The effect of a resonant $\nu_{e} \leftrightarrow \nu_{s}$ mixing on a type II supernovae was considered in [21]. The bounds on $\left(\Delta m_{e s}^{2}, \sin 2 \theta\right)$ derived in [21] are valid only if the sterile neutrinos have a mean free path larger than the radius of the supernova core after passing the resonance; this is the case if $\sin 2 \theta<3 \times 10^{-2}$. Our range is therefore unaffected by the type II supernovae constraint. On the other hand, a naive bound on the $\nu_{e} \leftrightarrow \nu_{s}$ mixing from big bang nucleosynthesis was derived, $\Delta m_{e s}^{2} \sin ^{4} 2 \theta \lesssim 5 \times 10^{-6} \mathrm{eV}^{2}$ [22]. Notice a disagreement of our preferred range with the above naive bound. However, the naive calculations ignored the creation of $\nu-\bar{\nu}$ asymmetries by active-sterile oscillations [23] in the early universe; these may efficiently suppress $\nu_{e} \leftrightarrow \nu_{s}$ oscillations, and therefore invalidate the conclusions drawn from naive calculations (even maximal $\nu_{\mu} \leftrightarrow \nu_{s}$ oscillations as a solution of the atmospheric neutrino anomaly 24] cannot be excluded 25]). The measurement of the flux of UHE neutrinos could thus provide us with a new test for this cosmological scenario.

To summarize, the dispersion relation for electron (anti)neutrinos in the Earth's interior for energies above the pole of the $W$ resonance, is derived. Then we have considered MSW oscillations for cosmic neutrinos traversing the Earth by including the charged-current self energy diagram for $\nu_{e}$. We have shown that the range of neutrino masses where maximal enhancement may occur could be interesting from a viewpoint of astrophysics and cosmology. Let us finally stress that in order to study a nadir angle dependence beyond $34^{\circ}$, where neutrinos always propagate outside the core, a weaker attenuation of a $\nu_{e}$ beam would require the inclusion of the tadpole self-energy for energies beyond $10^{9} \mathrm{GeV}$. This interesting case in now under study.

Acknowledgments. The author acknowledges the support of the Croatian Ministry of Science and Technology under the contract 1-03-068. 


\section{REFERENCES}

[1] R. Gandhi, C. Quigg, M. H. Reno and I. Sarcevic, Astropart. Phys. 5 (1996) 81.

[2] R. Gandhi, C. Quigg, M. H. Reno and I. Sarcevic, Phys. Rev. D 58 (1998) 093009.

[3] S. Iyer, M. H. Reno and I. Sarcevic, hep-ph/9909393.

[4] T. K. Gaisser, F. Halzen and T. Stanev, Phys. Rep. 258 (1995) 173.

[5] See e.g. F. W. Stecker, C. Done, M. H. Salamon and P. Sommers, Phys. Rev. Lett. 66 (1991) 2697; ibid. 69 (1992) 2738E; K. Mannheim, Astropart. Phys. 3 (1995).

[6] P. L. Anthony et al., Phys. Rev. Lett. 75 (1995) 1949.

[7] L. D. Landau and I. A. Pomeranchuk, Dokl. Akad. Nauk SSSR 92 (1953) 535; A. B. Migdal, Phys. Rev. 103 (1956) 1811.

[8] S. P. Mikheyev and A. Yu. Smirnov, Sov. J. Nucl. Phys. 42, (1985) 913; L. Wolfenstein, Phys. Rev. D 17 (1978) 2369.

[9] Q. Y. Liu and A. Yu. Smirnov, Nucl. Phys. B 524 (1998) 505; Q. Y. Liu, S. P. Mikheyev and A. Yu. Smirnov, Phys. Lett. B 440, (1998) 319; S. T. Petcov, Phys. Lett. B 434, (1998) 321; Eratum 444 (1998) 584; E. Kh. Akhmedov et al., Nucl. Phys. B 542 (1999) 3; M. V. Chizov, M. Maris and S. T. Petcov, hep-ph/9810501; S. T. Petcov, hepph/9907216; A. Yu. Smirnov, hep-ph/9809481, hep-ph/9811296, hep-ph/9907296; M. V. Chizov, hep-ph/9909439; A. Nicolaidis, G. Tsirigoti and J. Hansson, hep-ph/9904415.

[10] M. V. Chizov and S. T. Petcov, Phys. Rev. Lett. 83 (1999) 1096; M. V. Chizov and S. T. Petcov, hep-ph/9903424.

[11] See e.g. M. Le Bellac, Thermal Field Theory (Cambridge University Press, Cambridge, 1996).

[12] See e.g. I. E. McCarthy and E. Weigold, Rep. Prog. Phys. 54 (1991) 789.

[13] See e.g. L. D. Landau and E. M. Lifshitz, Course in Theoretical Physics Vol 3: Quantum Mechanics Non-relativistic Theory (Butterworth-Heinemann, 1981).

[14] F. D. Stacey, Physics of the Earth, 2nd ed. (Wiley, New York, 1977).

[15] See e.g. A. Kusenko, astro-ph/9903167 and references therein.

[16] S. Dodelson and L. M. Widrow, Phys. Rev. Lett. 72 (1994) 17; G. M. Fuller and X. Shi, astro-ph/9810076.

[17] C. Athanassopoulos et al., Phys. Rev. Lett. 77 (1996) 3082; ibid. 81 (1998) 1774.

[18] J. T. Peltoniemi and J. W. F. Valle, Nucl. Phys. B 406 (1993) 409; D. O. Caldwell and R. N. Mohapatra, Phys. Rev. D 48 (1993) 32.

[19] KARMEN Collab., K. Eitel, B. Zeitnitz, in Proceedings of Neutrino-98, Nucl. Phys. (Proc. Suppl.) 77 (1999) 212.

[20] T. K. Kuo and J. Pantaleone, Rev. Mod. Phys. 61 (1989) 937.

[21] X. Shi and G. Sigl, Phys. Lett. B 323 (1994) 360.

[22] R. Barbieri and A. Dolgov, Phys. Lett. B 237 (1990) 440; Nucl. Phys. B 237 (1991) 742; K. Kainulainen, Phys. Lett. B 244 (1990) 191; K. Enquist, K. Kainulainen and J. Maalampi, Phys. Lett. B 249 (1990) 531; Nucl. Phys. B 249 (1991) 754.

[23] R. Foot, M. Thompson and R. R. Volkas, Phys. Rev. D 53 (1996) 5349; R. Foot and R. R. Volkas, Phys. Rev. D 55 (1997) 5147; X. Shi and M. Fuller, Phys. Rev. D 59 (1999) 063006; P. Di Bari, P. Lipari and M. Lusignoli, hep-ph/9907548.

[24] M. C. Gonzalez-Garcia et al., Phys. Rev. D58 (1998) 33004; M. C. Gonzalez-Garcia et al., hep-ph/9807305; R. Foot, R. R. Volkas and O. Yasuda, Phys. Rev. D58 (1998) 013006; O. Yasuda, hep-ph/9809206. 
[25] R. Foot and R. R. Volkas, astro-ph/9811067. 